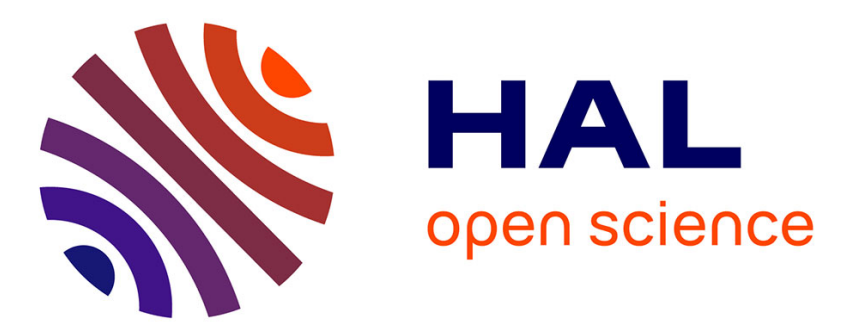

\title{
Easy distributions for combinatorial optimization problems with probabilistic constraints
}

\author{
Bernard Fortz, Michael Poss
}

\section{To cite this version:}

Bernard Fortz, Michael Poss. Easy distributions for combinatorial optimization problems with probabilistic constraints. Operations Research Letters, 2010, 38, pp.545-549. hal-01255270

\author{
HAL Id: hal-01255270 \\ https://hal.science/hal-01255270
}

Submitted on 30 Mar 2021

HAL is a multi-disciplinary open access archive for the deposit and dissemination of scientific research documents, whether they are published or not. The documents may come from teaching and research institutions in France or abroad, or from public or private research centers.
L'archive ouverte pluridisciplinaire HAL, est destinée au dépôt et à la diffusion de documents scientifiques de niveau recherche, publiés ou non, émanant des établissements d'enseignement et de recherche français ou étrangers, des laboratoires publics ou privés. 


\title{
Easy distributions for combinatorial optimization problems with probabilistic constraints
}

\author{
Bernard Fortz * $\quad$ Michael Poss ${ }^{\dagger}$
}

August 27, 2010

\begin{abstract}
We show how we can linearize individual probabilistic linear constraints with binary variables when all coefficients are independently distributed according to either $\mathcal{N}\left(\mu_{i}, \lambda \mu_{i}\right)$, for some $\lambda>0$ and $\mu_{i}>0$, or $\Gamma\left(k_{i}, \theta\right)$ for some $\theta>0$ and $k_{i}>0$. The constraint can also be linearized when the coefficients are independent and identically distributed and either positive or strictly stable random variables.
\end{abstract}

Keywords: Stochastic programming, combinatorial optimization, probabilistic constraint.

\section{Introduction}

Many combinatorial optimization models address problems with parameters which are impossible to predict exactly. Therefore, it is often more accurate to model these parameters with random variables. This modifies the structure of the optimization problems, depending on the times at which decisions are taken and parameters are revealed. In this note we study probabilistic constraints: all decisions must be taken here and now, such that the constraints of the model shall be satisfied with a certain probability. In other words, we aim at maximizing some objective for a given feasibility tolerance.

Stochastic programs with linear probabilistic constraints are in general non convex non linear optimization problems, see [18 among others. If furthermore some variables are integer, they become non convex Mixed Integer Non Linear Problems [15. Although probabilistic constraints have been widely studied for many years, see [17, 34, 37] and the references therein, papers on problems with integer variables are not very numerous. Among them, problems featuring joint probabilistic constraints with random right hand side have been studied by [7, 8, 9] who propose exact and heuristic branch-and-bound algorithms, [13] who study formulations and bounding procedures, 23] who develop

\footnotetext{
*Department of Computer Science, Faculté des Sciences Université Libre de Bruxelles, Brussels, Belgium. Email: bfortz@ulb.ac.be.

†Department of Computer Science, Faculté des Sciences, Université Libre de Bruxelles, Brussels, Belgium. Corresponding author. Email: mposs@ulb.ac.be.
}

a column-generation based algorithm for a supply chain management problem, and 35. who introduce the concepts of $p$-inefficiency and provide extensive computational results for the probabilistic set-covering problem studied in [8. All these works handle probabilistic constraints through the concept of $p$-efficient points introduced by [32, apart from [35] which uses $p$-inefficient points instead.

Herein, we consider problems where uncertainty affects both sides of the constraints. A branch-and-bound algorithm and heuristics for such problems have been proposed in [5, 6, and 21 studies valid inequalities for the problem with individual probabilistic constraints with uncertainty in both sides.

In what follows, we are particularly interested by the case of individual probabilistic constraints while the random variables follow particular continuous distributions, among which Gaussian distributions. Previous results in this direction assume that all random variables are normally distributed. In that case, the probabilistic constraints can be rewritten as quadratic constraints (see $[19,33,39]$ ), convex under some assumption on the confidence level 29]. If all variables are binary, the constraints can be further linearized using classical techniques [16. Further work extends the classical gaussian framework to the more general class of radial distributions [12. The authors show how a probabilistic constraint can be written as a second-order cone convex constraint. The latter constraint can be linearized as well when working with binary variables.

In this note, we always assume that coefficients are independent continuous random variables. We show that an individual linear probabilistic constraint with binary variables is equivalent to a linear constraint when all coefficients are distributed according to either $\mathcal{N}\left(\mu_{i}, \lambda \mu_{i}\right)$, for some $\lambda>0$ and $\mu_{i}>0$, or $\Gamma\left(k_{i}, \theta\right)$ for some $\theta>0$ and $k_{i}>0$. The constraint can also be linearized when the coefficients are independent and identically distributed, and either positive or strictly stable random variables.

The next section describes precisely the constraints studied herein. Then, in Section 3 we study the case of identically distributed random variables, while in Section 4 we study gaussian and gamma random variables. Finally, Section 5 illustrates our results on a multicommodity flow problem arising in telecommunications networks, and we conclude in Section 6 . 


\section{Studied constraints}

In the following we study mainly the following type of probabilistic constraints,

$$
\mathcal{C}_{1}(x)=P\left(\sum_{i=1}^{n} a_{i} x_{i} \leq b\right) \geq p,
$$

though our results extend easily to

$$
\mathcal{C}_{2}(x)=P\left(\sum_{i=1}^{n} a_{i} x_{i} \leq c_{1} y_{1}+b\right) \geq p,
$$

and

$$
\begin{array}{r}
\mathcal{C}_{3}(x)=P\left(\sum_{i=1}^{n} a_{i} x_{i} \leq \sum_{j=1}^{m} c_{j} y_{j}+b\right) \geq p \\
\sum_{j=1}^{m} y_{j} \leq 1,
\end{array}
$$

where $p \in(0,1), a_{i}$ are independent random variables, $c_{i}$ and $b$ are fixed coefficients. In addition, we always consider that $x_{i}, y_{j} \in\{0,1\}$, for $1 \leq i \leq n$ and $1 \leq j \leq m$. The first constraint (1) is the so-called knapsack constraint, which plays an important role in capacitated problems such as unsplittable multi-commodity flow and generalized assignment problems. The second constraint (2) appears when the choice of the capacitated facilities to be built is part of the decision: $b$ denotes the initial capacity and $c_{1}$ the capacity provided by the facility. Typical examples are network design and facility location problems. Finally, in many technical problems we must choose at most one out of a set of different facilities, for instance, different capacities for a new link to install in a telecommunication network. This is represented by (3).

In what follows, we say that two constraints $C_{1}(x) \geq 0$ and $C_{2}(x) \geq 0$ are equivalent, denoted by $C_{1}(x) \geq 0 \Leftrightarrow$ $C_{2}(x) \geq 0$, if the sets $\left\{x \in\{0,1\}^{n}\right.$ s.t. $\left.C_{1}(x) \geq 0\right\}$ and $\left\{x \in\{0,1\}^{n}\right.$ s.t. $\left.C_{2}(x) \geq 0\right\}$ are equal.

\section{Identically distributed vari- ables}

We first consider (1) for the simple example where $a_{i}$ are positive random variables identically distributed. Since $a_{i}$ are positive, we see that

$$
P\left(\sum_{i=1}^{m} a_{i} \leq b\right) \leq P\left(\sum_{i=1}^{m-1} a_{i} \leq b\right) .
$$

Thus, the number of $x_{i}$ that can be equal to 1 can certainly not exceed

$$
N(b)=\max _{1 \leq l \leq n}\left\{l \text { s.t. } P\left(\sum_{i=1}^{l} a_{i} \leq b\right) \geq p\right\} .
$$

Conversely, if some binary vector $x$ satisfies $\sum_{i=1}^{n} x_{i} \leq$ $N(b)$, then certainly $x$ satisfies (1) because $a_{i}$ are identically distributed. Then, considering (2), the previous reasoning holds with $N(b)$ for $y_{1}=0$, and with $N\left(b+c_{1}\right)$ for $y_{1}=1$. Finally, this reasoning extends to the pair of constraints (3), since at most one of the $y_{j}$ can be equal to 1 . We just proved the following:

Proposition 1. Consider $n$ independent identically distributed positive random variables $a_{i}, 1 \leq i \leq n$. Then, for $x_{i}, y_{j} \in\{0,1\}, 1 \leq i \leq n$ and $1 \leq j \leq m$, the following constraints are equivalent:

1. $\mathcal{C}_{1}(x) \geq p \Leftrightarrow \sum_{i=1}^{n} x_{i} \leq N(b)$

2. $\mathcal{C}_{2}(x) \geq p \Leftrightarrow \sum_{i=1}^{n} x_{i} \leq\left(N\left(b+c_{1}\right)-N(b)\right) y_{1}+N(b)$

3. If furthermore, $\sum_{j=1}^{m} y_{j} \leq 1$, then $\mathcal{C}_{3}(x) \geq p \Leftrightarrow$ $\sum_{i=1}^{n} x_{i} \leq \sum_{j=1}^{m}\left(N\left(b+c_{j}\right)-N(b)\right) y_{j}+N(b)$

with $N(r)$ defined in (5) for any real $r$.

In the following, we focus on results of type 1 . since 2 . and 3. can be deduced from 1. by the above arguments.

Remark that computing the value of $N(b)$ requires, in general, the solution of a multivariate integral that must be solved using efficient packages for numerical integration, see 33. For some distributions, this computational burden can be avoided. For instance, if all $a_{i}$ are uniformly distributed between 0 and 1 , their sum is distributed according to (see for instance [14])

$$
f(z)=\frac{1}{n !} \sum_{k=0}^{n+1}(-1)^{k}\left(\begin{array}{c}
n+1 \\
k
\end{array}\right)\left[(z-k)_{+}\right]^{n} .
$$

The uniform distributions are not the only distributions which sum up nicely. Stable distributions satisfy interesting summation properties too. Recall that if $a_{i}$ are $n$ independent copies of a stable random variable $a$, then for any constants $x_{i}$ the random variable $\sum_{i=1}^{n} x_{i} a_{i}$ has the same distribution as $v_{n} a+w_{n}$ with some constants $v_{n}=n^{1 / \alpha}$ for some $\alpha \in(0,2]$, and $w_{n}$. Moreover, $a$ is said strictly stable if $w_{n}=0$ in the relation above. For instance, the Levy distribution, with density function equal to $f(z ; c)=\sqrt{\frac{c}{2 \pi}} \frac{e^{-c / 2 x}}{x^{3 / 2}}$ for $z \geq 0$, is positive (satisfying the hypothesis of Proposition 1) and stable so that sums of such distributions are easy to compute. We refer to 27. for a good introduction to stable distributions.

In general, the support of stable distributions intersects negative reals. For instance Gaussian and Cauchy distributions always have negative tails. We show next that property (4) still holds for strictly stable distributions. By definition

$$
\sum_{i=1}^{n} a_{i} \sim n^{1 / \alpha} a_{1} \quad \alpha \in(0,2],
$$


so that

$$
P\left(\sum_{i=1}^{n} a_{i} \leq b\right)=P\left(n^{1 / \alpha} a_{1} \leq b\right)=P\left(a_{1} \leq b n^{-1 / \alpha}\right) .
$$

If $b \geq 0$, the function $n \mapsto b n^{-1 / \alpha}$ is non increasing, implying (4). We obtain the following:

Proposition 2. Consider $n$ independent identically distributed strictly stable random variables $a_{i}, 1 \leq i \leq n$, and $b \geq 0$. Then, if $x_{i} \in\{0,1\}$ for each $1 \leq i \leq n$, the following constraints are equivalent:

$$
\mathcal{C}_{1}(x) \geq p \Leftrightarrow \sum_{i=1}^{n} x_{i} \leq N(b)
$$

with $N(b)$ defined in (5).

An example of strictly stable distribution with $\alpha=$ 1 is the Cauchy distribution, with density function $f\left(z ; z_{0}, \gamma\right)=\frac{1}{\pi}\left(\frac{\gamma}{\left(z-z_{0}\right)^{2}+\gamma^{2}}\right)$ for some location parameter $z_{0} \in \mathbb{R}$ and scale parameter $\gamma>0$.

\section{Non identically distributed variables}

A well known stable distribution is the Gaussian distribution. In fact, for Gaussian and gamma random variables we are able to derive stronger results, allowing for the random variables to be distributed differently, as long as some regularity condition holds. Consider independent Gaussian random variables, $a_{i} \sim \mathcal{N}\left(\mu_{i}, \sigma_{i}^{2}\right)$, $1 \leq i \leq n$. Then, $\mathcal{C}_{1}(x) \geq p$ can be rewritten (see for instance [33])

$$
\sum_{i=1}^{n} \mu_{i} x_{i}+\Phi^{-1}(p) \sqrt{\sum_{i=1}^{n} \sigma_{i}^{2} x_{i}^{2}} \leq b,
$$

where $\Phi$ is the cumulative distribution of the standard Gaussian distribution $\mathcal{N}(0,1)$. When $x \in\{0,1\}^{n}$, (6) can be linearized introducing additional continuous variables, see [16]. However, these linearizations contain significantly more variables than the direct linearization from Proposition (3) below.

Proposition 3. Consider $n$ independent random variables $a_{i} \sim \mathcal{N}\left(\mu_{i}, \lambda \mu_{i}\right), 1 \leq i \leq n$, for $\lambda>0$ and $\mu_{i}>0$. Then, if $x_{i} \in\{0,1\}$ for each $1 \leq i \leq n$, the following constraints are equivalent:

$$
\mathcal{C}_{1}(x) \geq p \Leftrightarrow \sum_{i=1}^{n} \mu_{i} x_{i} \leq \mu^{*}
$$

where $\mu^{*}$ is the unique root of the equation $b-\mu=$ $\Phi^{-1}(p) \sqrt{\lambda \mu}$.

Proof. Recall that if $a_{1}, \ldots, a_{n}$ are independent Gaussian with mean $\mu_{i}$ and variance $\sigma_{i}^{2}$, and $x_{i}$ are real numbers, then $a:=\sum_{i=1}^{n} x_{i} a_{i} \sim \mathcal{N}\left(\mu(x), \sigma^{2}(x)\right)$, with $\mu(x)=\sum_{i=1}^{n} x_{i} \mu_{i}$ and $\sigma^{2}(x)=\sum_{i=1}^{n} x_{i}^{2} \sigma_{i}^{2}$. Thus, because $x_{i} \in\{0,1\}$ and $\sigma_{i}^{2}=\lambda \mu_{i}$ for each $1 \leq i \leq n$, we have $\sigma^{2}(x)=\lambda \mu(x)$. Then,

$$
P\left(\sum_{i=1}^{n} a_{i} x_{i} \leq b\right)=P\left(\mathcal{N}(0,1) \leq \frac{b-\mu(x)}{\sqrt{\lambda \mu(x)}}\right),
$$

so that $\mathcal{C}_{1}(x) \geq p$ is equivalent to

$$
\frac{b-\mu(x)}{\sqrt{\lambda \mu(x)}} \geq \Phi^{-1}(p) .
$$

The left hand side of (8) is decreasing in $\mu(x)$, and thus $\mathcal{C}_{1}(x) \geq p$ is equivalent to $\mu(x) \leq \mu^{*}$, where $\mu^{*}$ is the unique root of the equation $b-\mu=\Phi^{-1}(p) \sqrt{\lambda \mu}$.

We provide in Section 5 an application of Proposition 3 to a routing problem arising in telecommunications. Similar examples can be devised for the generalized assignment problem, see for instance the Proportional Mean-Variance Model from 38, which assumes that random variables are those from Proposition 3 .

The next proposition considers the case of independent gamma random variables used, for instance, to model waiting and processing times in servers locations problems [10].

Proposition 4. Consider $n$ independent random variables $a_{i} \sim \Gamma\left(k_{i}, \theta\right), 1 \leq i \leq n$, for some $\theta>0$ and $k_{i}>0$, and assume that $b>0$. Then, if $x_{i} \in\{0,1\}$ for each $1 \leq i \leq n$, the following constraints are equivalent:

$$
\mathcal{C}_{1}(x) \geq p \Leftrightarrow \sum_{i=1}^{n} k_{i} x_{i} \leq k^{*}
$$

where $k^{*}$ is the unique solution of $\frac{\int_{0}^{b} z^{k-1} e^{\frac{-z}{\theta}} d z}{\Gamma(k) \theta^{k}}=p$ and the gamma function is defined by $\Gamma(k)=\frac{\int_{0}^{\infty} z^{k-1} e^{\frac{-z}{\theta}} d z}{\theta^{k}}$.

Proof. Gamma distributions satisfy also some kind of summation property, although weaker than the property satisfied by Gaussian distributions. Recall that if $a_{1}, \ldots, a_{n}$ are independent Gamma with shape $k_{i}$ and a common scale $\theta$, then $a:=\sum_{i=1}^{n} a_{i} \sim \Gamma(k, \theta)$, with $k=\sum_{i=1}^{n} k_{i}$. Thus, if $x_{i}$ are binary numbers, we have also that $a:=\sum_{i=1}^{n} x_{i} a_{i} \sim \Gamma(k(x), \theta)$, with $k(x)=\sum_{i=1}^{n} k_{i} x_{i}$. Thus, for binary $x_{i}, \mathcal{C}_{1}(x)$ is equivalent to $P(\Gamma(k(x), \theta) \leq b)$ defined by

$$
\frac{\int_{0}^{b} z^{k(x)-1} e^{\frac{-z}{\theta}} d z}{\Gamma(k(x)) \theta^{k(x)}},
$$

which we note $\mathcal{K}(k(x))$ in the following. Assuming that $\mathcal{K}(k)$ is a strictly decreasing function of $k$, the constraint $\mathcal{K}(k(x)) \geq p$ is equivalent to the constraint $k(x) \leq k^{*}$, with $k^{*}=\mathcal{K}^{-1}(p)$ which proves $\mathcal{C}_{1}(x) \geq p \Leftrightarrow$ $\sum_{i=1}^{n} k_{i} x_{i} \leq k^{*}$. Note that $\mathcal{K}^{-1}$ is well defined for any $p \in(0,1)$ because $\mathcal{K}$ is continuous, strictly decreasing, $\lim _{k \rightarrow 0^{+}} \mathcal{K}(k)=1$ and $\lim _{k \rightarrow+\infty} \mathcal{K}(k)=0$. 
We are left to prove that $\mathcal{K}(k)$ is a strictly decreasing function of $k>0$ :

$$
\begin{aligned}
\frac{d \mathcal{K}}{d k}(k)= & \theta \frac{d}{d k} \frac{\int_{0}^{b} z^{k-1} e^{-z} d z}{\int_{0}^{\infty} v^{k-1} e^{-v} d v} \\
= & \frac{\theta}{\Gamma^{2}(k)}\left(\int_{0}^{b} \ln (z) z^{k-1} e^{-z} d z \int_{0}^{\infty} v^{k-1} e^{-v} d v\right. \\
& \left.-\int_{0}^{b} z^{k-1} e^{-z} d z \int_{0}^{\infty} \ln (v) v^{k-1} e^{-v} d v\right) \\
= & \frac{\theta}{\Gamma^{2}(k)} \int_{0}^{b} d z \int_{b}^{\infty} d v\left(z^{k-1} v^{k-1} e^{-z-v} \ln \frac{z}{v}\right),
\end{aligned}
$$

which is strictly negative because $\ln \frac{z}{v}<0$ for $(z, v) \in$ $[0, b] \times(b, \infty)$.

When $b \leq 0, \mathcal{K}(k(x))=0$ so that the probabilistic constraint is equivalent to $\sum_{i=1}^{n} x_{i} \leq 0$.

\section{Application to the bandwidth packing problem}

In what follows, we apply Proposition 3 to a multicommodity flow problem occurring in telecommunications networks. We discuss different approaches to tackle the probabilistic constraints. Notice that our example is easily extended to the problem of designing a telecommunications network, replacing the fixed capacity $C$ by a set of facilities with capacity $C^{j}, j=1, \ldots, m$ as in (3).

\subsection{Problem description}

Given a directed graph $G=(V, A)$ with a capacity vector $C$, and a set of commodities $K$ of size $d^{k}$ and revenue $c^{k}$ from $s(k)$ to $t(k)$ for each $k \in K$, the bandwidth packing problem $(B W P)$ aims at routing commodities on the network in order to maximize the total revenue. For technical reasons based on routing protocols, each commodity must be sent along a unique path from $s(k)$ to $t(k)$, see [4, 30. Introducing the binary variable $x_{a}^{k}$ stating whether commodity $k$ is routed through $\operatorname{arc} a$, the problem can be formulated as

$$
\begin{aligned}
& \max \sum_{k \in K} \sum_{a \in A^{-}(t(k))} c^{k} x_{a}^{k} \\
& \text { s.t. } \quad \sum_{a \in A^{-}(v)} x_{a}^{k}-\sum_{a \in A^{+}(v)} x_{a}^{k}= \begin{cases}1 & v=t(k) \\
-1 & v=s(k) \\
0 & \text { else }\end{cases} \\
& k \in K, v \in V \\
& \sum_{k \in K} d^{k} x_{a}^{k} \leq C_{a} \\
& x_{a}^{k} \in\{0,1\} \\
& a \in A
\end{aligned}
$$

where $A^{+}(v)$ and $A^{-}(v)$ denote the set of outgoing arcs and incoming arcs at node $v$, respectively. In practice, although the traffic size $d^{k}$ varies along time, it is not convenient to change the routing according tho these variations; $x$ must be set once for a given time period. Different frameworks allow to model such uncertainties. Some works consider that $d$ belongs to a polyhedron $\mathcal{D}$ and that (9) must be feasible for any $d \in \mathcal{D}$, see [22] and the closely related 2, among others. Others [31] model $d^{k}, k \in K$, by random variables and replace 9 by

$$
P\left(\sum_{k \in K} d^{k} x_{a}^{k} \leq C_{a}\right) \geq p \quad a \in A
$$

In what follows, we assume that $d^{k}, k \in K$, are independent Gaussian distributed according to $\mathcal{N}\left(\mu^{k}, \lambda \mu^{k}\right)$. The Gaussian assumption has been studied in [1, 20] and used in [3], among others. Moreover, 36] (followed by 24] and [3) assume that $d^{k}$ and $d^{h}$ are independently distributed for $k \neq h$. Finally, some authors [26] suggest that means and variances are linearly correlated as traffic size increases, that is, $\sigma=\lambda \mu$ for some $\lambda>0$, so that we can apply Proposition 3 to 10 .

\subsection{Solution methods}

We review different approaches to tackle the chanceconstrained version of $(B W P)$. Besides Proposition 3 there are two groups of methods to handle $(10)$. Keeping the random vector continuous, we can tackle $(10)$ by MINLP methods. Alternatively, we can sample the random variables to obtain a scenario set $S$ and solve the deterministic equivalent.

Direct linearization We apply Proposition 3 to 100 , obtaining again problem $(B W P)$ with $d^{k}$ and $C_{a}$ replaced by $\mu^{k}$ and the unique root $\mu_{a}^{*}$ of $C_{a}-\mu=$ $\Phi^{-1}(p) \sqrt{\lambda \mu}$, respectively. Computing $\mu_{a}^{*}$ is easy since function $\frac{C_{a}-\mu}{\sqrt{\lambda \mu}}$ is convex and differentiable. Therefore, we can solve the problem with efficient algorithms used in the deterministic case, such as the branch-and-cutand-price algorithm from [4.

MINLP methods When $p \geq 0.5$ and each $d^{k}$ is Gaussian, (10) is convex and thus, well suited for non linear algorithms [11. However, it is clearly easier to use the direct linearization of (10) through Proposition 3 , because non-linear constraints are harder to handle than linear ones and both formulations provide the same bound. For instance, outer approximation-based algorithms replace 10) by a set of tangent cutting planes. The latter contains more inequalities, with possibly highly fractional coefficients, than the unique inequality resulting from 10.

Alternatively, 10 with Gaussian random variables can be reformulated as (6). We can then rewrite (6) 
as

$$
\begin{aligned}
& \sum_{k \in K} \mu^{k} x_{a}^{k} \leq C_{a} \\
& \sum_{k \in K}\left[\left(\Phi^{-1}(p) \sigma^{k}\right)^{2}+\mu^{k}\left(2 C_{a}-\mu^{k}\right)\right] x_{a}^{k} \\
& +\sum_{k, h \in K: k \neq h}\left[\left(\left(\Phi^{-1}(p)\right)^{2} \sigma^{k} \sigma^{h}-2 \mu^{k} \mu^{h}\right)\right] x_{a}^{k} x_{a}^{h} \leq C_{a}^{2},
\end{aligned}
$$

for each $a \in A$. When $p>0.5$, which is the case in real situations, $\mu_{a}^{*}<C_{a}$ and thus, 111) is less tight than (7). Hence, Proposition (10) allows to strengthen the above formulation by substituting (11) with (7). Then, (12) is not needed anymore to define a valid formulation. However, since it takes into account the binary restriction on $x$ (by using $\left(x_{a}^{k}\right)^{2}=x_{a}^{k}$ ), it may be used together with (7) to provide a stronger continuous relaxation. Note finally that linearizing (12) requires at least $|K|$ additional variables and $2|K|$ additional constraints for each $a \in A[16$.

Discretization and deterministic equivalent Sampling a scenario set $S$ that approximates the continuous distribution $d$ in an acceptable way, see [25, 28], among others, we can write a deterministic equivalent for 10 :

$$
\begin{array}{lr}
\sum_{k \in K} d^{k s} x_{a}^{k} \leq C_{a}+M_{a}^{s} y_{a}^{s} & a \in A, s \in S \\
\sum_{s \in S} p^{s} y_{a}^{s} \geq p & a \in A,
\end{array}
$$

where components of vector $M$ are numbers large enough. However, (13) and (14) yield a very difficult problem because (13) contains a large number of constraints and features "big-M" coefficients. Therefore, [5, 6] show how to replace (13) and (14) by a relevant set $L$ of scenario sets through a branch-and-bound algorithm. Each $l \in L$ yields a problem similar to $(B W P)$, but with multiple capacity constraints 9 for each arc $a \in A$ (one for each scenario in $l$ ). Then, using bounding mechanisms, they avoid solving all problems associated to elements of $L$. Eventually, the exact approaches from [5. 6] will have solved several binary multi-commodity flow problems with multiple capacity constraints, each of them being more more complex than $(B W P)$. Although applicable to a broader class of problems, this approach will in general be slower than the direct linearization from Proposition 3 that requires only to solve one problem similar to $(B W P)$ plus the computation of the root vector $\mu^{*}$.

\section{Conclusion}

In this note we show how to linearize individual probabilistic constraints with binary variables under very specific assumptions. Then, we propose a model for the bandwidth packing problem with probabilistic capacity constraints under demand uncertainty that satisfies our assumptions, and discuss different approaches to tackle the problem. We show that our linearization method simplifies considerably the stochastic problem because the latter becomes as easy to solve as its deterministic version. We can thus apply efficient algorithms taking the combinatorial structure of the problem into account.

In opposition, recent solution methods to integer programs featuring probabilistic constraints with uncertainty in both sides, such as [5, 6], are far more general and only assume that the uncertain parameters are described by a finite scenario set. Nevertheless, these approaches require to solve integer programs significantly harder than the deterministic versions.

\section{Acknowledgements}

This research is supported by an "Actions de Recherche Concertées" (ARC) projet of the "Communautée française de Belgique". Michael Poss is a research fellow of the "Fonds pour la Formation à la Recherche dans l'Industrie et dans l'Agriculture" (FRIA). The authors would also like to thank a referee for constructive criticism.

\section{References}

[1] F. Alagöz, Approximations on the aggregate mpeg video traffic and their impact on admission control, Turk. J. Elec. Eng. 10 (2002), no. 1, 73-84.

[2] A. Altin, E. Amaldi, P. Belotti, and M. Ç. Pinar, Provisioning virtual private networks under traffic uncertainty, Networks 49 (2007), no. 1, 100-115.

[3] R. Andrade, A. Lisser, N. Maculan, and G. Plateau, Telecommunication network capacity design for uncertain demand, Comput. Optim. Appl. 29 (2004), no. 2, 127-146.

[4] C. Barnhart, C. A. Hane, and P. H. Vance, Using branch-andprice-and-cut to solve origin-destination integer multicommodity flow problems, Oper. Res. 48 (2000), no. 2, 318-326.

[5] P. Beraldi and M. E. Bruni, A probabilistic model applied to emergency service vehicle location, European Journal of Operational Research 196 (2009), no. 1, 323-331.

[6] _ An exact approach for solving integer problem under probabilistic constraints with random technology matrix, Annals of Operations Research 177 (2010), 127-137.

[7] P. Beraldi and A. Ruszczynski, A branch and bound method for stochastic integer problems under probabilistic constraints, Optimization Methods and Software 17 (2002), 359-382.

[8], The probabilistic set-covering problem, Oper. Res. 50 (2002), no. 6, 956-967.

[9] , Beam search heuristic to solve stochastic integer problems under probabilistic constraints, European Journal of Operational Research 167 (2005), no. 1, 35-47.

[10] O. Berman and D. Krass, Facility location problems with stochastic demands and congestion, In: Drezner, Z. and Hamacher, H.W. (eds.) Facility location: applications and theory, ch. 11, pp. 329-371, Springer, 2004.

[11] P. Bonami, M. Kilinc, and J. Linderoth, Algorithms and software for convex mixed integer nonlinear programs, Tech. Report 1664, Computer Sciences Department, University of WisconsinMadison, 2009. 
[12] G. Calafiore and L. El Ghaoui, Distributionally robust chanceconstrained linear programs with applications, J. of Optimization Theory and Applications 130 (2006), no. 1, 1-22.

[13] D. Dentcheva, A. Prékopa, and A. Ruszczynski, Bounds for probabilistic integer programming problems, Discrete Applied Mathematics 124 (2002), no. 1-3, 55 - 65.

[14] C. Grinstead and J. Snell, Introduction to probability, 2nd edn, American Math. Society, Providence, 1997.

[15] I. E. Grossmann, Review of nonlinear mixed-integer and disjunctive programming techniques, Optimization and Engineering 3 (2002), 227-252(26)

[16] P. Hansen and C. Meyer, Improved compact linearizations for the unconstrained quadratic 0-1 minimization problem, Discrete Applied Mathematics 157 (2009), 1267-1290.

[17] R. Henrion, Introduction to chance-constrained programming, Tutorial paper for the Stochastic Programming Community Home Page, 2004

[18] R. Henrion and C. Strugarek, Convexity of chance constraints with independent random variables, Comput. Optim. Appl. 41 (2008), 263-276.

[19] S. Kataoka, A stochastic programming model, Econometrica 31 (1963), no. $1 / 2,181-196$.

[20] J. Kilpi and I. Norros, Testing the gaussian approximation of aggregate traffic, IMW '02: Proceedings of the 2nd ACM SIGCOMM Workshop on Internet measurment (New York, NY, USA), ACM, 2002, pp. 49-61.

[21] O. Klopfenstein, Solving chance-constrained combinatorial problems to optimality. Computational Optimization and Applications 45 (2010), no. 3, 607-638.

[22] O. Klopfenstein and D. Nace, Valid inequalities for a robust knapsack polyhedron - application to the robust bandwidth packing problem, Networks (2010), to appear.

[23] M. A. Lejeune and A. Ruszczynski, An efficient trajectory method for probabilistic production-inventory-distribution problems, Operations Research 55 (2007), no. 2, 378-394.

[24] A. Lisser, A. Ouorou, J.-P. Vial, and J. Gondzio, Capacity planning under uncertain demand in telecommunication networks, Tech. report, 1999

[25] J. Luedtke and S. Ahmed, A sample approximation approach for optimization with probabilistic constraints, SIAM Journal on Optimization 19 (2008), no. 2, 674-699.

[26] R. Morris and D. Lin, Variance of aggregated web traffic, Proceedings of INFOCOM, 2000, pp. 360-366.

[27] J. P. Nolan, Stable distributions - models for heavy tailed data, Birkhäuser, Boston, 2010, In progress, Chapter 1 online at academic2.american.edu/ jpnolan.

[28] B. Pagnoncelli, S. Ahmed, and A. Shapiro, Sample average ap proximation method for chance constrained programming: Theory and applications, Journal of Optimization Theory and Applications 142 (2009), 399-416.

[29] S. Parikh, Lecture notes on stochastic programming, University of California, Berkeley, CA, 1968.

[30] K. Park, S. Kang, and S. Park, An integer programming approach to the bandwidth packing problem, Manage. Sci. 42 (1996), no. 9, 1277-1291.

[31] F. Pascali, Chance constrained network design, Ph.D. thesis, Universitá di Pisa, 2009.

[32] A. Prékopa, Dual method for a one-stage stochastic programming with random rhs obeying a discrete probability distribution, Zeitschrift Oper. Res. 34 (1990), 441--461.

[33] _ Stochastic programming, Kluwer, 1995.

[34] _ Probabilistic programming models, In: Ruszczynski, A., Shapiro, A. (eds.) Stochastic Programming: Handbook in Operations Research and Management Science, vol. 10, ch. 5, pp. 267351, Elsevier Science Ltd, Amsterdam, 2003.
[35] A. Saxena, V. Goyal, and M. A. Lejeune, MIP reformulations of the probabilistic set covering problem, Math. Program. 121 (2009), no. 1, 1-31.

[36] S. Sen, R. D. Doverspike, and S. Cosares, Network planning with random demand, Telecommunication Systems 3 (1994), no. 1, 11-30.

[37] A. Shapiro, D. Dentcheva, and A. Ruszczynski, Lectures on stochastic programming: Modeling and theory, SIAM, Philadelphia, 2009.

[38] D. Spoerl and R.K Wood, A stochastic generalized assignment problem, INFORMS Annual Meeting, Atlanta, GA, 19-22 October, 2003.

[39] C. van de Panne and W. Popp, Minimum-cost cattle feed under probabilistic protein constraints, Management Science 9 (1963), no. 3, 405-430. 